\title{
Information Retrieval Based Nearest Neighbor Classification for Fine-Grained Bug Severity Prediction
}

\author{
Yuan Tian ${ }^{1}$, David $\mathrm{Lo}^{1}$, and Chengnian $\mathrm{Sun}^{2}$ \\ ${ }^{1}$ Singapore Management University, Singapore \\ ${ }^{2}$ National University of Singapore, Singapore \\ \{yuan.tian.2012,davidlo\}@smu.edu.sg, suncn@comp.nus.edu.sg
}

\begin{abstract}
Bugs are prevalent in software systems. Some bugs are critical and need to be fixed right away, whereas others are minor and their fixes could be postponed until resources are available. In this work, we propose a new approach leveraging information retrieval, in particular BM25-based document similarity function, to automatically predict the severity of bug reports. Our approach automatically analyzes bug reports reported in the past along with their assigned severity labels, and recommends severity labels to newly reported bug reports. Duplicate bug reports are utilized to determine what bug report features, be it textual, ordinal, or categorical, are important. We focus on predicting fine-grained severity labels, namely the different severity labels of Bugzilla including: blocker, critical, major, minor, and trivial. Compared to the existing state-of-the-art study on fine-grained severity prediction, namely the work by Menzies and Marcus, our approach brings significant improvement.
\end{abstract}

\section{INTRODUCTION}

Software systems usually contain defects that need to be fixed after releases, and in some projects users are allowed to feedback on these defects that they encounter through bug reporting systems such as Bugzilla. With Bugzilla, users can report not only the description of the bug but also estimate the severity of the reported bugs. Unfortunately, although guidelines exist on how severity of bugs need to be assigned, the process is inherently manual that is highly dependent on the expertise of the bug reporters in assigning correct labels. Novice bug reporter might find it difficult to decide the right severity level. Developers (aka. Bugzilla assignee) can later adjust the severity [1] and use this severity information to prioritize which bugs to be fixed first.

As the number of bug reports made is large, a number of past studies have proposed approaches to help users in assigning severity labels, and development team in validating bug report severity [18], [14], [15]. All these approaches combine text processing with machine learning to assign severity labels from the textual description of the reports. Menzies and Marcus develop a machine learning approach to assign the severity labels of bug reports in NASA [18]. More recently, Lamkanfi et al. develop another machine learning approach to assign severity labels of bug reports in several Bugzilla repositories of open source projects [14]. In a later work, Lamkanfi et al. have also tried many different classification algorithms and investigate their effectiveness in assigning severity labels to bug reports [15]. Menzies and Marcus assign fine-grained labels (5 severity labels used in NASA), while Lamkanfi et al. assign coarse-grained labels (i.e., binary labels: severe and non-severe).

The bug severity prediction tools are not perfect though and there is still room for improvement. Menzies and Marcus reported $\mathrm{F}$ measures (i.e., harmonic mean of precision and recall) of 14 to $86 \%$ for the different severity labels [18]. Lamkanfi et al. reported $\mathrm{F}$ measures of $65 \%$ to $75 \%$ on Bugzilla reports from different software systems [14]. Thus there is a need to improve the accuracy of the prediction tools further.

In this work, we propose an information retrieval (IR)based nearest neighbor solution to predict the severity labels of bug reports. We first measure the similarity of different bug reports and based on this similarity we recover past bug reports that are most similar to it. There are various measures that have been proposed in the information retrieval community to measure the similarity between two textual documents [22], [31], [27], [26]. Some of the popular techniques are BM25 and its extensions [26]. BM25 technique and its extensions require some parameters to be learned. We leverage bug reports that have been marked as duplicate to set these parameters. Our hypothesis is that duplicate bug reports would help us to identify what features are important and what are not to measure the similarity between two bug reports. Based on a set of $k$ nearest neighbors, the severity labels of these $k$ similar bug reports are then used to decide the appropriate severity label for a new bug report.

In this work, we focus on predicting fine-grained bug severity labels. We investigate the effectiveness of our proposed approach and compare it with the past study by Menzies and Marcus [18]. Since our approach requires duplicate bug reports, we do not use the NASA data investigated by Menzies and Marcus. Rather, we analyze a large number of bug reports stored in Bugzilla bug tracking systems of Eclipse, OpenOffice, and Mozilla. We focus on predicting five severity labels of Bugzilla namely: blocker, critical, major, minor, and trivial. Following the work of Lamkanfi et al. [14], [15], we do not consider the severity label normal as this is the default option and "many 
reports just did not bother to consciously assess the bug severity" [14], [15]. Thus, we treat these reports as unlabeled data.

Our experiments show that we could achieve a precision, recall, and $\mathrm{F}$ measure of up to $72 \%, 76 \%$, and $74 \%$ for predicting a particular class of severity labels. Precision quantifies the amount of false positives, while recall quantifies the amount of false negatives. High precision and high recall mean less number of false positives and less number of false negatives respectively. $F$ measure is the harmonic mean of precision and recall. Comparing with the stateof-the-art work on fine-grained severity level prediction by Menzies and Marcus, we show that for most bugs and most severity labels we could improve their approach significantly, especially on hard-to-predict ${ }^{1}$ severity labels.

The following lists our contributions:

1) We propose an information retrieval based nearest neighbor solution, by leveraging duplicate bug reports, to predict fine-grained severity labels.

2) We have experimented our solution and compare it with the state-of-the-art work over a collection of more than 65,000 bug reports from three medium-large software systems: OpenOffice, Mozilla, and Eclipse.

3) We show that we can achieve a significant improvement over the state-of-the-art fine-grained bug severity prediction technique, especially on hard-to-predict severity labels.

The structure of this paper is as follows. In Section II, we describe some background material related to bug reporting and text pre-processing. In Section III, we elaborate our approach. We present our experiments and their results in Section IV. We discuss related work in Section V. We conclude and describe future work in Section VI.

\section{BACKGROUND}

In this section, we describe the bug reporting process, then present standard approaches to pre-process textual documents, and finally highlight $B M 25 F_{\text {ext }}$ to measure the similarity between structured documents.

\section{A. Bug Reporting}

To help improve the quality of software systems, software projects often allow users to report bugs. This is true for both open-source and closed-source software developments. Bug tracking systems such as Bugzilla are often used. Users from various locations can log in to Bugzilla and report new bugs. Users can report symptoms of the bugs along with other related information to developers. These include textual descriptions of the bug either in short or detailed form, product and component that are affected by the bug, and the estimated severity of the bug. The format of bug

\footnotetext{
${ }^{1} \mathrm{~F}$ measures of these labels are much lower than the others.
}

reports varies from one project to another, but bug reports typically contain the fields described in Table I.

Developers (in particular bug triagers) would then verify these symptoms and fix the bugs. They could make adjustment to the severity of the reported bug. There are often many reports that are received and thus developers would need to prioritize which reports are more important than others - the severity field is useful for this purpose. As bug reporting is a distributed process, often the same bug is reported by more than two people in separate bug reports. This is known as duplicate bug report problem. The developer/triager would also need to identify these duplicate bug reports so as not to waste bug fixing efforts.

\section{B. Text Pre-Processing}

Tokenization. A token is a string of characters, and includes no delimiters such as spaces, punctuation marks, and so forth. Tokenization is the process of parsing a character stream into a sequence of tokens by splitting the stream at delimiters.

Stop-Word Removal. Stop words are non-descriptive words carrying little useful information for retrieval tasks. These include linking verbs such as "is", "am" and "are", pronouns such as "I", "he" and "it", etc. Our stop word list contains 30 stop words, and also common abbreviations such as "I'm", "that's", "we'll", etc..

Stemming. Stemming is a technique to normalize words to their ground forms. For example, a stemmer can reduce both "working" and "worked" to "work". This better allows a machine learning algorithm to capture the similarity between two or more words. We used Porter stemming algorithm [25] to process our text.

\section{BM25F and Its Extension}

We present $B M 25 F$, and $B M 25 F_{\text {ext }}$. The first is a standard document similarity function, the latter is the extended $\mathrm{BM} 25 \mathrm{~F}$ proposed in [26] to handle longer query documents.

BM25F Similarity Function. BM25F is a function to evaluate the similarity between two structured documents [21], [32]. A document is structured if it has a number of fields. A bug report is a structured document as it has several textual fields, i.e., summary and description. Each of the fields in the structured document can be assigned a different weight to denote its importance in measuring the similarity between two documents.

Before we proceed further, let's define a few notations. Consider a document corpus $D$ consisting of $N$ documents. Also, each document $d$ has $K$ fields. Let's denote the bag of words in the $f^{t h}$ field as $d[f]$ for $1 \leq f \leq K$.

BM25F similarity function has two primary components which assign global and local importance to words. The global importance of a word $t$ is based on its inverse document frequency (IDF). This IDF score is inversely 
Table I

FIELDS OF INTEREST IN A BUG REPORT

\begin{tabular}{|c|c|}
\hline Field & Description \\
\hline Summ & Summary: Short description of the bug which typically contains only but a few words. \\
\hline Desc & $\begin{array}{l}\text { Description: Detailed description of the bug. This includes information such as how to } \\
\text { reproduce the bug, the error log outputted when the bug occurs, etc. }\end{array}$ \\
\hline Prod & Product: Product that is affected by the bug. \\
\hline Comp & Component: Component that is affected by the bug. \\
\hline Sev & $\begin{array}{l}\text { Severity: Estimated impact of the bug to the workings of the software. In Bugzilla, there are } \\
\text { several severity levels: blocker, critical, major, normal, minor, and trivial. } \\
\text { There is also another severity level, enhancement which we ignore in this work, as we } \\
\text { are not interested in feature requests but only defects. }\end{array}$ \\
\hline
\end{tabular}

Table II

EXAMPlES OF BUg REPORTS FROM MOZILla BUGZILlA

\begin{tabular}{|c|c|c|c|c|c|}
\hline & ID & Summary & Product & Component & Severity \\
\hline \multirow[t]{2}{*}{1} & 525359 & $\begin{array}{l}\text { replying to an HTML message which includes a contenteditable div leaves } \\
\text { Thunderbird compose unusable until restart (from incredimail for example) }\end{array}$ & Thunderbird & $\begin{array}{l}\text { Message } \\
\text { Compose } \\
\text { Window }\end{array}$ & major \\
\hline & 543032 & $\begin{array}{l}\text { Impossible to answer a mail from thunderbird } 3.01 \text { after viewing an e-mail } \\
\text { sent by Incredimail }\end{array}$ & Thunderbird & $\begin{array}{l}\text { Message } \\
\text { Compose } \\
\text { Window }\end{array}$ & critical \\
\hline \multirow[t]{2}{*}{2} & 537897 & No way to select engines when setting up to use an existing account & Mozilla Services & $\begin{array}{l}\text { Firefox } \\
\text { Backend }\end{array}$ & normal \\
\hline & 543686 & Everything is synced when logging in to an existing account & Mozilla Services & Firefox Sync, UI & normal \\
\hline \multirow[t]{2}{*}{3} & 538953 & $\begin{array}{l}\text { Using Search bar AND a proxy with password authentification ... keeps } \\
\text { asking the password at any key entered }\end{array}$ & Firefox & Search & normal \\
\hline & 544836 & Proxy authentication broken while typing in the search field & Firefox & Search & major \\
\hline
\end{tabular}

proportional to the number of documents containing a word; it is defined in Equation 1.

$$
I D F(t)=\log \frac{N}{N_{t}}
$$

In Equation (1), $N_{t}$ is the number of documents containing the word $t$.

Another component prescribes the local importance of a word $t$ in a document $d$. This local importance, denoted as $T F_{D}(d, t)$, is defined in Equation 2. This is the aggregation of the local importance of the word $t$ for each of document $d$ 's field.

$$
T F_{D}(d, t)=\sum_{f=1}^{K} \frac{w_{f} \times \text { occurrences }(d[f], t)}{1-b_{f}+\frac{b_{f} \times \text { size }_{f}}{\text { avg_size }_{f}}}
$$

In Equation (2), $w_{f}$ is the weight of field $f$, occurrences $(d[f], t)$ is the number of times the word $t$ occurs in field $f$, size $_{f}$ is the number of words in $d[f]$, avg_size $f$ is the average size of $d[f]$ for all documents in $D$, and $b_{f}$, which takes the value between 0 to 1 , is a parameter that controls the contribution of the size of the fields to the overall score.

Based on the global and local term importance weights, given two documents $d$ and $q$, each of which is a bag of words, the BM25F score of $d$ and $q$ is:

$$
\operatorname{BM25F}(d, q)=\sum_{t \in d \cap q} I D F(t) \times \frac{T F_{D}(d, t)}{k+T F_{D}(d, t)}
$$

In Equation (3), the word $t$ is common in $d$ and $q$, and $k$, whose value is greater or equal to zero, is a parameter that controls the contribution of $T F_{D}(d, t)$ to the overall score. We notice that BM25F has a number of free parameters that need to be tuned: $w_{f}$ and $b_{f}$ for each document's field, and $k$. Given a document containing $K$ fields, BM25F requires $(1+2 K)$ parameters to be tuned. An optimization technique based on stochastic gradient descent has been used to tune these BM25F parameters [30].

$B M 25 F_{\text {ext }}$ Similarity Function. BM25F is particularly developed to compute similarity of a short document (i.e., query) with a longer document. It is typically used for search engines, where user queries are usually short and consist of only a few words. However, bug reports are longer textual documents - the description field of a bug report can contain a few hundred words. Thus, since we want to have a similarity function that measures the similarity of two bug reports each of which are relatively long textual documents, there is a need to extend BM25F. Sun et al. [26] address this need by proposing $B M 25 F_{\text {ext }}$ which considers the term frequencies in queries; it has the following form.

$$
\begin{array}{r}
B M 25 F_{\text {ext }}(d, q)=\sum_{t \in d \cap q} I D F(t) \times \frac{T F_{D}(d, t)}{k+T F_{D}(d, t)} \times W_{Q} \\
\text { where } W_{Q}=\frac{(l+1) \times T F_{Q}(q, t)}{l+T F_{Q}(q, t)}
\end{array}
$$




$$
T F_{Q}(q, t)=\sum_{f=1}^{K} w_{f} \times \text { occurrences }(q[f], t)
$$

In Equation (4), for each common word $t$ appearing in document $d$ and query $q$, its contribution to the overall $B M 25 F_{\text {ext }}$ score has two components: one is the product of $I D F$ and $T F_{D}$ inherited from $B M 25 F$; and the other is the local importance of word $t$ in the document $q-$ denoted as $W_{Q} . W_{Q}$ follows the word weighting scheme of Okapi BM25 [16]. Parameter $l$, whose value is always greater than or equal to 0 , controls the contribution of the local importance of word $t$ in $q$ to the overall score - if $l=0$, then the local importance of $t$ in $q$ is ignored, and $B M 25 F_{\text {ext }}$ becomes BM25F.

In Equation (5), the contribution of each word $t$ is the summation of the product of $w_{f}$, which is the weight of field $f$, with the number of occurrences of $t$ in the $f^{t h}$ field of $q$. Different from $T F_{D}$, defined in Equation 2, to compute $T F_{Q}$, we do not perform any normalization. We do not perform normalization as retrieval is being done with respect to a single fixed query - we want to rank bug reports based on their similarities to a given query bug report.

$B M 25 F_{\text {ext }}$ requires an additional free parameter $l$ in addition to those needed by $B M 25 F$. This brings the total numbers of parameters for $B M 25 F$ to $(2+2 K)$. These parameters can be set by following a gradient descent approach presented in [26].

\section{PROPOSED APPROACH}

In this section, we describe our proposed approach. We first summarize our approach. We then highlight two major components of our approach.

\section{A. Overall Framework}

Our framework assigns a severity label to a bug report $B Q$ in question by investigating prior bug reports with known severity labels in the pool of bug reports BPool. The high-level pseudocode of our approach, named IR Based

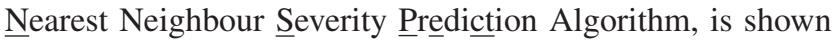
in Figure 1. The algorithm would first find the top-k nearest neighbors (Line 1) and then predict the label by considering the labels of these nearest neighbors (Lines 2-3).

Our framework thus consists of two major components: similarity computation, which is an integral part of finding nearest neighbors, and label assignment. In the similarity computation component, we measure the similarity between two bug reports. We leverage duplicate bug reports as training data to assign features that are important to measure how similar two reports are. We use an extended BM25 document similarity measure for the purpose. In the label assignment component, given a bug report whose severity is to be predicted, we take the nearest $k$ bug reports based on the similarity measure. These $k$ bug reports are then used to predict the label of the bug report.

\section{Procedure INSPect}

Inputs:

$B Q$ : Bug report in question

BPool: Historical bug report pool

Output: Predicted bug report severity label

Methods:

1: Let NNSet $=$ Find top-K nearest neighbors of $B Q$ in $B P$ Pool 2: Let PredictedLabel $=$ Predict label from NNSet

3: Output PredictedLabel

Figure 1. IR Based Nearest Neighbour Severity Prediction Algorithm

\section{B. Similarity Computation}

A bug report contains more than textual features, it also contains other information such as product, component, etc. We want to make use of all these features, textual and nontextual, to detect the similarity among bug reports. To do this, given two bug reports $d$ and $q$, our similarity function $\operatorname{REP}(d, q)$ is a linear combination of four features, with the following form where $w_{i}$ is the weight for the $i$-th feature feature $_{i}$.

$$
\operatorname{REP}(d, q)=\sum_{i=1}^{4} w_{i} \times \text { feature }_{i}
$$

Each weight determines the relative contribution and the degree of importance of its corresponding feature. Features that are important to measure the similarity between bug reports would have a higher score. Each of the four features along with their definitions are given in Figure 2. There are two types of features: textual and non-textual; we elaborate them in the following paragraphs.

Textual Features. The first feature of Equation (7) is the textual similarity of two bug reports based on the summary and description fields as measured by $B M 25 F_{\text {ext }}$ similarity function described in Section II. The second feature is similar to the first one, except that summary and description fields are represented by bags of bigrams (a bigram is two words that appear consecutively one after the other) instead of bags of words (or unigrams).

Non-Textual Features. The other two features have binary values ( 0 or 1$)$ based on the equality of the product and component fields of $d$ and $q$.

The similarity function $R E P$ defined in Equation (6) has 16 free parameters in total. For feature $_{1}$ and feature $_{2}$, we compute textual similarities of $d$ and $q$ over two fields: summary and description. Computing each of the two features requires $(2+2 \times 2)=6$ free parameters. Also, we need to weigh the contributions of each of the 4 features in Equation (6). Thus overall, $R E P$ requires $(2 \times 6+4)=16$ parameters to be set. Table III lists all these parameters.

The above metric is similar to the one proposed by Sun et al. [26] except we remove several features: one is a binary feature that compares the types of the reports: defect, 


$$
\begin{aligned}
& \text { feature }_{1}(d, q)=B M 25 F_{\text {ext }}(d, q) / / \text { of unigrams } \\
& \text { feature }_{2}(d, q)=B M 25 F_{\text {ext }}(d, q) / / \text { of bigrams } \\
& \text { feature }_{3}(d, q)= \begin{cases}1, & \text { if } d \cdot p r o d=q . p r o d \\
0, & \text { otherwise }\end{cases} \\
& \text { feature }_{4}(d, q)= \begin{cases}1, & \text { if } d . c o m p=q . \operatorname{comp} \\
0, & \text { otherwise }\end{cases}
\end{aligned}
$$

\begin{tabular}{|c|c|}
\hline Parameter & Description \\
\hline$\overline{w_{1}}$ & weight of feature ${ }_{1}$ (unigram) \\
\hline$w_{2}$ & weight of feature $_{2}$ (bigram) \\
\hline$w_{3}$ & weight of feature fproduct) $_{3}$ \\
\hline$w_{4}$ & weight of feature $_{4}$ (component) \\
\hline$w_{\text {summ }}^{\text {unigram }}$ & "weight of summary in feature ${ }_{1}$ \\
\hline$w_{\text {desc }}^{\text {unigram }}$ & weight of description in feature ${ }_{1}$ \\
\hline$b_{\text {summ }}^{\text {unigram }}$ & $b$ of summary in feature 1 \\
\hline$b_{\text {desc }}^{\text {unigram }}$ & $b$ of description in feature $_{1}$ \\
\hline$k_{1}^{\text {unigram }}$ & $k_{1}$ in feature $_{1}$ \\
\hline$k_{3}^{\text {unıgram }}$ & $k_{3}$ in feature $_{1}$ \\
\hline$\overline{w_{\text {summ }}^{\text {bigram }}}$ & "weight of summary in feature ${ }_{2}$ \\
\hline$w_{\text {desc }}^{\text {bigram }}$ & weight of description in feature $_{2}$ \\
\hline$b_{\text {summ }}^{\text {biescim }}$ & $b$ of summary in feature $_{2}$ \\
\hline$b_{\text {desc }}^{\text {bigram }}$ & $b$ of description in feature f $_{2}$ \\
\hline$k_{1}^{\text {bigram }}$ & $k_{1}$ in feature $_{2}$ \\
\hline$k_{3}^{\text {bigram }}$ & $k_{3}$ in feature $_{2}$ \\
\hline
\end{tabular}

Figure 2. Features in the Retrieval Function

Table III

PARAMETERS IN $R E P$

enhancement, etc., another is a feature that computes the difference between the reported severities, and the other is a feature that computes the difference between the versions. Since we only consider defects, and we assume that severity label is not available, we could not use the first two of the three omitted features to compute similarity between bug reports. We do not use the last feature as we do not have the complete version information for all subject programs which requires manual crawling of the web. $R E P$ parameters are tuned using gradient descent. We take a training set consisting of duplicate bug reports, and follow the same approach as proposed in the work by Sun et al. [26]. We include the above description to ensure that our paper is self-explanatory.

\section{Label Assignment}

Leveraging the similarity measure, we locate the top$\mathrm{k}$ nearest neighbors of a bug report in question. We then aggregate the contribution of each bug report to predict the label of the bug report. We compute the weighted mean of the labels of the neighbors as the predicted label. We map the labels into integers and order them from the most severe to the least severe. The labels blocker, critical, major normal, minor, and trivial are mapped to $0,1,2,3$, 4 , and 5 respectively.

Consider a set of nearest neighbors NNSet of a bug report $B Q$. Also let $N N S e t[i]$ be the ith nearest neighbor, NNSet[i].Label be the label of the ith nearest neighbor (expressed in integer), and NNSet [i].Sim be the similarity of $B Q$ with $N N S e t[i]$. The predicted label is computed by the following formula:

$$
\left\lfloor\frac{\sum_{i=0}^{k}(N N S e t[i] . S i m \times N N \text { Set }[i] . \text { Label })}{\sum_{i=0}^{k}(N N S e t[i] . \text { Sim })}+0.5\right\rfloor
$$

The above formula aggregates the label of each neighbor based on its similarity with the target bug report $B Q$. The higher is a neighbor similarity with $B Q$, the more powerful it is in influencing the label of $B Q$. The formula ensures that the label would fall into the range. We use the floor operation and the " +0.5 " to round the resultant label to the nearest integer.

As bug reports with normal severity are treated as unlabeled data, we ignore the contributions of these reports. In case the $k$ neighbors of a new bug report whose severity label is to be predicted are all assigned normal label, we simply assign label major to the new bug report.

Example. To illustrate the above, we present an example.

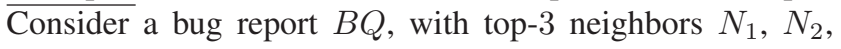
and $N_{3}$ with labels 5,4 , and 3 respectively. Let the $R E P$ similarity scores of $B Q$ with each of the neighbors to be:

$$
\begin{aligned}
& R E P\left(B Q, N_{1}\right)=0.5 \\
& R E P\left(B Q, N_{2}\right)=0.45 \\
& R E P\left(B Q, N_{3}\right)=0.35
\end{aligned}
$$

The assigned label of $B Q$ would then be:

$$
\begin{aligned}
& =\left\lfloor\frac{\sum_{i=0}^{3}\left(R E P\left(B Q, N_{i}\right) \times N_{i} . \text { Label }\right)}{\sum_{i=0}^{k}\left(R E P\left(B Q, N_{i}\right)\right)}+0.5\right\rfloor \\
& =\left\lfloor\frac{(0.5 \times 5+0.45 \times 4+0.35 \times 3)}{(0.5+0.45+0.35)}+0.5\right\rfloor \\
& =\left\lfloor\frac{(2.5+1.8+1.05)}{1.3}+0.5\right\rfloor \\
& =4
\end{aligned}
$$

\section{EXPERIMENTS}

In this section, we highlight the datasets that we use in this study, followed by our experimental settings. We then present the measures used to evaluate the approaches, followed by our results. Finally, we also mention some threats to validity.

\section{A. Datasets}

We chose the bug repositories of three large open source projects: OpenOffice, Mozilla and Eclipse, as the three 
projects have different backgrounds, implementation languages and users, which can help generalizing the conclusions of our experiments. OpenOffice is a multi-platform and multi-lingual office suite. Mozilla is a not-for-profit community producing open-source software and technologies used by other applications, such as the Firefox browser and Rhino JavaScript interpreter. Eclipse is a large project aiming to build a flexible development platform for all lifecycles of software development.

We extract three datasets from the open source projects by collecting reports submitted within a period of time. Each dataset only contains defect reports, whereas feature requests and maintenance tasks are filtered away. We use the final assigned severity labels in the defect reports as the ground truth. Table IV details the three datasets. We construct a training set by selecting the first $M$ reports of which 200 reports are duplicates, in order to tune the parameters in the retrieval function $R E P$, regardless of the size of the resultant bug report set for training. Those $M$ reports are also used to simulate the initial bug repository for all experimental runs. The number $M$ for the 3 datasets are given in sub-column \# All of column Training Reports in Table IV. The rest of the reports are used for testing the prediction approach, shown in column Testing Reports.

\section{B. Experimental Settings}

We propose an online evaluation approach that mimics how severity prediction could be used in practice. At each experimental run, we iterate through the reports in the set of testing reports in chronological order. Once we reach a report $R$, we apply a severity prediction tool to predict the severity label of $R$. This would be the recommendation given to the user/developer on the severity of the bug report. As the accuracy of all existing severity prediction techniques are still low, humans/developers/triagers cannot be completely taken out from the picture. At the beginning of the next iteration, we add $R$ and its true label (we assume triagers make the right decision and give a correct feedback) to the pool of bug reports BPool in Figure 1. After the last iteration is done, we measure how good the recommendations are. Similar online evaluation approaches have been used in evaluating studies on the detection of duplicate bug reports [27] and on the recommendation of developers to fix bug reports [29].

Unfortunately, the classification based approaches employed in [18] (i.e., Severis) is slow. For around 4,000 bug reports of OpenOffice, employing the online evaluation approach would mean re-training the classification model for around 4,000 times - we re-train the model everytime a new user feedback is received. This took us more than 10 hours. As the number of bug reports increases the runtime increase in a super-linear fashion as at each step in the online evaluation approach more bug reports need to be investigated to train the model. Thus, we also evaluate the existing approach in an offline manner - we take a set of bug reports that we use to train REP to train Severis. We then use trained Severis to assign labels to the remaining set of bug reports.

We perform both offline and online evaluation for Severis on OpenOffice bugs. We show that the results of these two evaluation approaches do not differ much. We only perform offline evaluation for Severis for the other two bug report datasets: Mozilla, and Eclipse. As our approach is fast and relies on nearest neighbors, we only do the online strategy.

\section{Evaluation Measures}

We use the standard measures of precision, recall, and $\mathrm{F}$ measure for each severity label to evaluate the effectiveness of Severis and INSPect. F measure is the harmonic mean of precision and recall and it is often used to measure if an increase in precision/recall outweighs a loss in recall/precision. The same measures were used by Menzies and Marcus to evaluate Severis [18]. The definitions of precision, recall, and $\mathrm{F}$ measure for a severity label $S^{L}$ are given below ${ }^{2}$ :

$$
\operatorname{precision}\left(S^{L}\right)=\frac{\# \text { reports correctly labeled with } S^{L}}{\# \text { reports labeled with } S^{L}}
$$

$\operatorname{recall}\left(S^{L}\right)=\frac{\# \text { reports correctly labeled with } S^{L}}{\# \text { reports that should be labeled with } S^{L}}$

$$
F \operatorname{Measure}\left(S^{L}\right)=2 \times \frac{\operatorname{precision}\left(S^{L}\right) \times \operatorname{recall}\left(S^{L}\right)}{\operatorname{precision}\left(S^{L}\right)+\operatorname{recall}\left(S^{L}\right)}
$$

\section{Comparison Results}

We compare INSPect with parameter $k$ set to 1 (i.e., 1-nearest neighbor) and Severis on the three datasets. We present the results in the following sub-sections.

1) OpenOffice Results: The result of INSPect on bug reports of OpenOffice is shown in Table V. Different from the other three programs in OpenOffice there are only five severity levels [19]. We map them to critical, major, normal, minor, and trivial. Again we drop normal from our analysis. We note that we can predict the critical, major, minor, and trivial severity labels by $\mathrm{F}$ measures of $36.0 \%, 74.0 \%, 39.8 \%$, and $22.2 \%$ respectively. The F measure is very good for ma jor severity label but is poorest for trivial severity label.

The result for Severis (offline) is also shown in Table V. We note that Severis can predict the critical, major, minor, and trivial severity labels by $\mathrm{F}$ measures of $25.6 \%, 75.1 \%, 20.5 \%$, and $1.2 \%$ respectively. Comparing these with the result of INSPect, we note that INSPect can improve the $\mathrm{F}$ measure for critical, minor, and trivial labels by a relative improvement of $41 \%, 94 \%$,

\footnotetext{
${ }^{2}$ \# reports refers to number of reports.
} 
Table IV

DETAILS OF DATASETS

\begin{tabular}{|c|c|c|c|c|c|c|c|c|}
\hline \multirow{2}{*}{ Dataset } & \multicolumn{2}{|c|}{ Period } & \multicolumn{3}{c|}{ Training Reports } & \multicolumn{3}{c|}{ Testing Reports } \\
\cline { 2 - 9 } & From & To & \#Duplicate & \#All & \#All - \#Normal & \#Duplicate & \#All & \#All - \#Normal \\
\hline OpenOffice & $2008-01-02$ & $2010-12-21$ & 200 & 2,986 & 617 & 488 & 20,438 & 3,356 \\
\hline Mozilla & $2010-01-01$ & $2010-12-31$ & 200 & 4,379 & 1,273 & 1,802 & 68,049 & 16,490 \\
\hline Eclipse & $2001-10-10$ & $2007-12-14$ & 200 & 3,312 & 500 & 6,203 & 175,297 & 43,587 \\
\hline
\end{tabular}

Table V

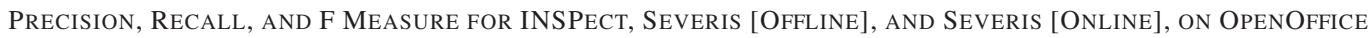

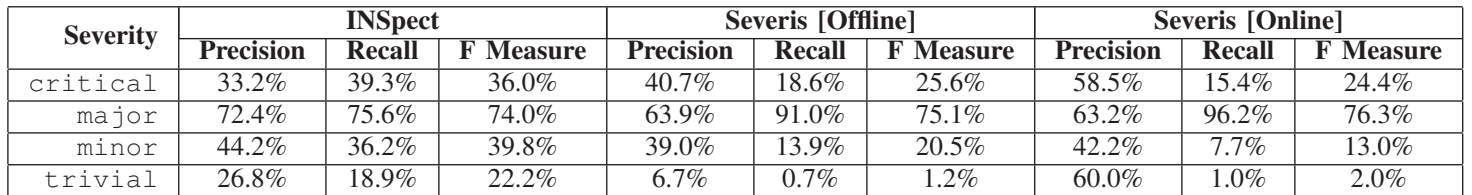

and $1748 \%$ respectively. For the ma jor label, INSPect lose out to Severis by only $2 \%$. Thus for OpenOffice, in general our proposed approach INSPect performs better than Severis.

Although expensive (in terms of runtime; it takes more than 10 hours to complete), we also run Severis using the online evaluation approach and present the result in Table V. We notice that the result using the online evaluation, although requires much more computation time, generally is not better than using offline evaluation. There is a small increase in F measure for major and trivial; However, for critical and minor there is a small reduction in $\mathrm{F}$ measure.

2) Mozilla Results: The result of INSPect on bug reports of Mozilla is shown in Table VI. We note that we can predict the blocker, critical, major, minor, and trivial severity labels by $\mathrm{F}$ measures of $32.6 \%, 65.9 \%$, $54.3 \%, 35.9 \%$, and $35.8 \%$ respectively. The $\mathrm{F}$ measure is very good for critical severity label but is poorest for blocker severity label.

The result for Severis is also shown in Table VI. Note that we only run the offline version of Severis as the online version takes much time, and our experiment with OpenOffice shows that employing online or offline evaluation does not affect the performance of Severis. We note that Severis can predict the blocker, critical, major, minor, and trivial severity labels by $\mathrm{F}$ measures of $0.4 \%$, $65.1 \%, 59.7 \%, 3.4 \%$, and $2.2 \%$ respectively. Comparing these with the result of INSPect, we note that we can improve the F measures for blocker, critical, minor, and trivial labels by a relative improvement of $8,038 \%$, $1.2 \%, 957 \%$, and $1,528 \%$ respectively. For the ma jor label, INSPect lose out to Severis by $9.0 \%$. Thus for Mozilla, in general our proposed approach INSPect performs better than Severis.

3) Eclipse Results: The result of INSPect on bug reports of Eclipse is shown in Table VII. We note that we can predict the blocker, critical, major, minor, and trivial severity labels by $\mathrm{F}$ measures of $26.0 \%, 29.0 \%, 57.8 \%$,
$40.3 \%$, and $26.5 \%$ respectively. The $\mathrm{F}$ measure is very good for major severity label but is poorest for blocker severity label.

The result for Severis is also shown in Table VII. We note that Severis can predict the blocker, critical, major, minor, and trivial severity labels by $\mathrm{F}$ measures of $0.0 \%, 28.5 \%, 56.0 \%, 0.2 \%$, and $0.0 \%$ respectively. The F measures of Severis are zeros for blocker and trivial as it does not assign any bug report to those severity labels. Comparing these with the result of INSPect, we note that we can improve the $\mathrm{F}$ measure for blocker, critical, major, minor, and trivial labels by a relative improvement of infinity, $1.7 \%, 3.2 \%, 20,055 \%$, and infinity, respectively. INSPect does not lose out to Severis for any label. Thus for Eclipse, clearly INSPect performs better than Severis.

\section{E. Varying parameter $k$}

Our proposed approach INSPect takes in one user defined parameter $k$. In the previous experiments we set $k$ to 1 . We want to investigate the effect of changing the parameter $k$ on the overall effectiveness of our solution. We plot the effect of varying $k(k=1,5,10,20)$ on $\mathrm{F}$ measure for OpenOffice, Mozilla, and Eclipse datasets in Figures 3, 4, \& 5 respectively. When we increase $k$, we consider more nearest neighbors. This might increase accuracy as in effect we are tapping more to the "wisdom of the masses". However, this might also reduce accuracy as the additional neighbors might not be that similar anymore to the target bug report.

From the figures, for OpenOffice, the $\mathrm{F}$ measure of critical increases as we increase $k$. However, the $\mathrm{F}$ measures of minor and trivial decrease as we increase $k$. For Mozilla, the F measure of ma jor slightly increases as we increase $k$, however, for three severity labels, blocker, minor, and trivial, their $\mathrm{F}$ measures decrease as we increase $k$. For Eclipse, the $\mathrm{F}$ measure of critical increases as we increase $k$. However, the $\mathrm{F}$ measures of three severity labels, blocker, minor and trivial decrease 
Table VI

Precision, Recall, and F Measure For INSPect and SEVEris [OFFline] on Mozilla

\begin{tabular}{|r|c|c|c|c|c|c|}
\hline \multirow{2}{*}{ Severity } & \multicolumn{3}{|c|}{ INSpect } & \multicolumn{3}{c|}{ Severis [Offline] } \\
\cline { 2 - 7 } & Precision & Recall & F Measure & Precision & Recall & F Measure \\
\hline blocker & $33.9 \%$ & $31.3 \%$ & $32.6 \%$ & $100 \%$ & $0.2 \%$ & $0.4 \%$ \\
\hline critical & $64.0 \%$ & $67.8 \%$ & $65.9 \%$ & $82.6 \%$ & $53.7 \%$ & $65.1 \%$ \\
\hline major & $53.5 \%$ & $55.2 \%$ & $54.3 \%$ & $43.9 \%$ & $93.1 \%$ & $59.7 \%$ \\
\hline minor & $38.9 \%$ & $33.4 \%$ & $35.9 \%$ & $50.5 \%$ & $1.8 \%$ & $3.4 \%$ \\
\hline trivial & $38.4 \%$ & $33.6 \%$ & $35.8 \%$ & $19.7 \%$ & $1.1 \%$ & $2.2 \%$ \\
\hline
\end{tabular}

Table VII

Precision, Recall, and F Measure for INSPECt And SEVEris [OfFline] on Eclipse

\begin{tabular}{|r|c|c|c|c|c|c|}
\hline \multirow{2}{*}{ Severity } & \multicolumn{3}{|c|}{ INSpect } & \multicolumn{3}{c|}{ Severis [Offline] } \\
\cline { 2 - 7 } & Precision & Recall & F Measure & Precision & Recall & F Measure \\
\hline blocker & $25.2 \%$ & $27.0 \%$ & $26.0 \%$ & $0.0 \%$ & $0.0 \%$ & $0.0 \%$ \\
\hline critical & $28.2 \%$ & $29.8 \%$ & $29.0 \%$ & $22.3 \%$ & $39.7 \%$ & $28.5 \%$ \\
\hline major & $58.0 \%$ & $57.5 \%$ & $57.8 \%$ & $48.2 \%$ & $66.8 \%$ & $56.0 \%$ \\
\hline minor & $42.4 \%$ & $38.4 \%$ & $40.3 \%$ & $7.6 \%$ & $0.1 \%$ & $0.2 \%$ \\
\hline trivial & $28.2 \%$ & $25.0 \%$ & $26.5 \%$ & $0.0 \%$ & $0.0 \%$ & $0.0 \%$ \\
\hline
\end{tabular}

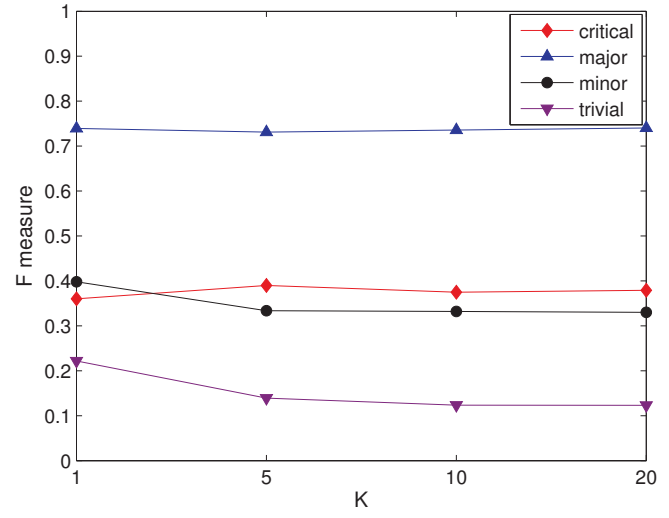

Figure 3. OpenOffice: Varying $k$ and Its Effect on F Measure

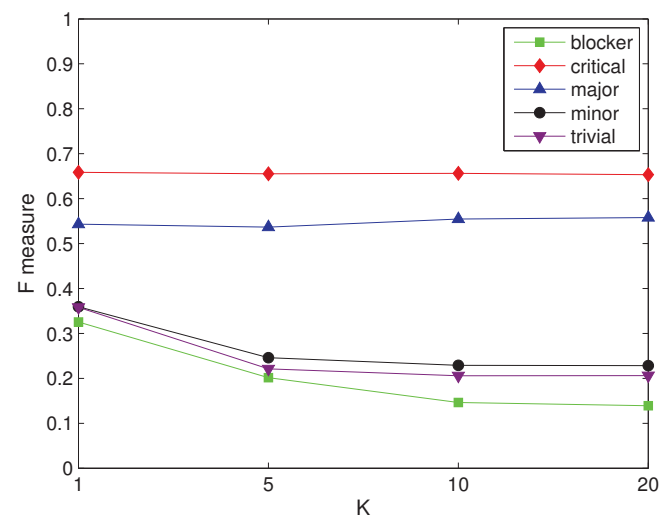

Figure 4. Mozilla: Varying $k$ and Its Effect on F Measure

as we increase $k$.

\section{F. Threats to Validity \& Discussion}

We consider three threats of validity: threats to construct validity, threats of internal validity, and threats of external validity.

Threats to construct validity relates to the suitability of

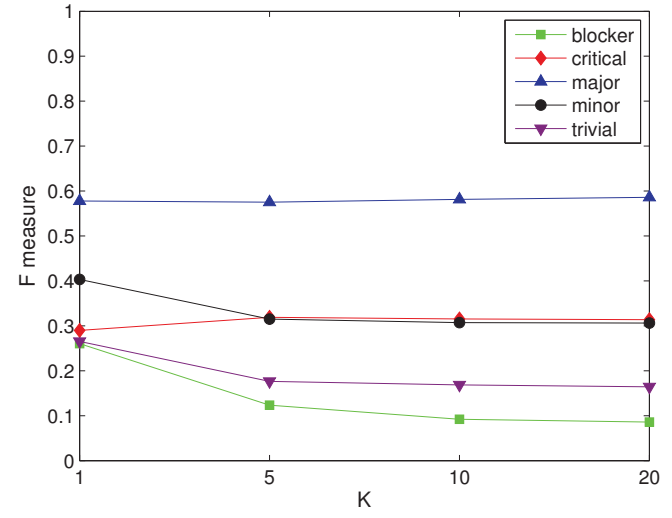

Figure 5. Eclipse: Varying $k$ and Its Effect on F Measure

our evaluation metrics. We use standard metrics used in classification and prediction namely: precision, recall, and $\mathrm{F}$ measure. These measures have been used before by Menzies and Marcus to evaluate Severis [18].

Threats of internal validity refers to errors in our experiments. We extract the severity labels from the various Bugzilla bug tracking systems. We assume that except the normal label, the severity labels recorded in Bugzilla are the final severity labels that are deemed correct. We use these ground truth labels to measure how good our predictions are. A similar assumption and experimental setting were also made in prior studies [14], [15].

Threats of external validity refers to the generalizability of our findings. We consider repositories of three mediumlarge software systems: Eclipse, OpenOffice, and Mozilla. We consider a total of more than 65,000 bug reports. This is larger than the number of bug reports considered in prior studies [18], [14], [15]. Furthermore, the three projects are written in different programming languages, and have different background and user groups. Also, all our studies make use of open source repositories where data is publicly 
available. We do not use the datasets from NASA used in [18] and made available in the Promise repository as they do not have information on duplicate bug reports. Note that duplicate bug reports are common phenomenon, and many of them are found in many open source Bugzilla tracking systems, c.f., [22], [31], [13], [27], [26].

\section{RELATED WORK}

In this section, we highlight related studies on bug severity prediction, bug report analysis, and text mining for software engineering.

\section{A. Past Studies on Bug Severity Prediction}

There are a number of studies that predict the severity of bug reports [15], [14], [18]. We highlight these studies in the following paragraphs.

Menzies and Marcus predict the severity of bug reports in NASA [18]. They first extract word tokens from bug reports, and then perform stop word removal and stemming. Important tokens are then identified using the concept of term frequency-inverse document frequency, and information gain. These tokens are then used as features for a classification approach named Ripper rule learner [6]. Their approach is able to identify fine grained bug report labels, which are the the 5 severity levels used in NASA.

More recently, Lamkanfi et al. predict the severity of bug reports from various projects' Bugzilla bug tracking systems [14]. They first extract word tokens and pre-process them. These tokens are then fed to a Naive Bayes classifier to predict the severity of the corresponding bug. Different from the work by Menzies and Marcus, they predict coarse grained bug severity labels: severe, and non-severe. Three of the six classes of severity in Bugzilla (blocker, critical, and major) are grouped as severe, two of the six classes (minor, and trivial) are grouped as nonsevere, and normal severity bugs are omitted from their analysis.

Extending the above work, Lamkanfi et al. also try out various classification algorithms to predict the severity of bug reports [15]. They show that Naive Bayes performs better than other mining approaches on a dataset of 29,204 bug reports.

Our approach extends the above research studies. Similar to Menzies and Marcus's work, we detect fine grained bug report labels. Similar to the work by Lamkanfi et al. we consider bug reports on Bugzilla repositories of various open source projects. We compare our approach with that of Menzies and Marcus on a dataset containing more than 65,000 bug reports and show that we could gain significant $\mathrm{F}$ measure improvements.

\section{B. Other Studies Analyzing Bug Reports}

In a related research area, recently a number of techniques are proposed for duplicate bug report retrieval [22], [31],
[13], [27], [26]. Many of these approaches propose various ways to measure the similarity of bug reports to help developers in assigning bug reports as either duplicate or not. Runeson et al. propose a formula that considers the frequency of common words appearing in both documents as a similarity measure [22]. Wang et al. use both term frequency and inverse document frequency as a similarity measure [31]. They also consider a special situation where runtime traces are available and could be used to compute the similarity between bug reports. In practice, however, only a small minority of bug reports come with runtime traces. Jalbert and Weimer propose yet another term frequency based similarity measure [13]. Sun et al. propose a technique that leverages SVM for duplicate bug report detection [27]. In their later work, Sun et al. propose an approach to measuring the similarity of bug reports using an enhanced BM25F document similarity measure [26]. These recent advances in bug report similarity measurement could potentially be leveraged to categorize bug reports into various severity classes. Our work shows that they are indeed useful for this purpose.

Another line of research is categorization of bug reports to reduce maintenance effort. Anvik et al. [2], Cubranic and Murphy [7], Tamrawi et al. [28] propose various techniques to automatically assign the right developer for a new report. Huang et al. categorize bug reports into those related to capability, security, performance, reliability, requirement, and usability [12]. Pordguski et al. [20] and Francis et al. [8] propose approaches to group reported software failures, by analyzing the corresponding execution traces. Gegick et al. identify security bug reports using text mining [9]. The approach to some extent is similar to the work of Lamkanfi et al. [14], in that it categorizes bugs into two categories. However rather than categorizing bug into: severe and notsevere, it categorizes bug into: security-related and nonsecurity-related.

Previous work also conducts empirical studies on bug repositories. Anvik et al. study the characteristics of bug repositories and show interesting findings on the number of reports that a person submit and the proportion of various resolutions [3]. Sandusky et al. study the nature, impact and extent of a bug report network in one large open source development community[23]. Hooimeijer and Weimer predict the quality of bug reports by a novel descriptive model built based on surface features of over 27,000 bug reports from several open source projects [11]. Bettenburg et al. describe characteristics of good bug reports by surveying Eclipse, Mozilla and Apache developers [4].

\section{Text Mining for Software Engineering}

There are many studies that utilize various forms of text analysis and mining for software engineering purposes. Haiduc et al. propose a method to summarize source code to support program comprehension [10]. The work proposes 
an approach to extract informative yet succinct text to characterize source code entities so that developers can better understand a large piece of code. Sridhara et al. propose an approach to detect code fragments implementing high level abstractions and describe them in succinct textual descriptions [24].

Marcus and Maletic propose an approach to link documentation to source code using Latent Semantic Indexing [17]. Chen et al. proposed an approach to link textual documents to source code by combining several techniques including regular expression, key phrases, clustering and vector space model [5].

Similar to the above studies, we also extend a text mining approach to solve problem in software engineering. Different from the above studies, we investigate a different problem namely the prediction of fine-grained bug report severity label from its text. Our approach combines nearest neighbor classification and an extension of BM25 document similarity function.

\section{CONCLUSION AND Future WORK}

Severity labels are important for developers to prioritize bugs. A number of existing approaches have been proposed to infer these labels from textual fields of bug reports. In this work, we propose a new approach to infer severity labels from various information available from bug reports: textual, and non-textual. We make use of duplicate bug reports to weigh the relative importance of each piece of information or features to determine the similarity between bug reports. This similarity measure is then used in a nearest-neighbor fashion to assign a severity label to a bug report. We have compared our approach to the state-of-the-art approach on fine-grained severity label prediction, namely Severis. Extensive experiments on tens of thousands of bug reports taken from three medium-large software systems: Eclipse, OpenOffice, and Mozilla, have been performed. The result shows that we can improve the $\mathrm{F}$ measure of the state-ofthe-art approach significantly, especially on hard-to-predict severity labels.

As future work, we plan to improve the accuracy of the proposed approach further. We also plan to embed our solution into Bugzilla to let it be used by many people.

\section{REFERENCES}

[1] http://wiki.eclipse.org/WTP/Conventions_of _bug_priority_and_severity\#How_to_set_Severity_and_Priority.

[2] J. Anvik, L. Hiew, and G. Murphy, "Who should fix this bug?" in proceedings of the International Conference on Software Engineering, 2006.

[3] J. Anvik, L. Hiew, and G. C. Murphy, "Coping with an open bug repository," in ETX, 2005, pp. 35-39.

[4] N. Bettenburg, S. Just, A. Schröter, C. Weiss, R. Premraj, and T. Zimmermann, "What makes a good bug report?" in SIGSOFT FSE, 2008, pp. 308-318.

[5] X. Chen and J. C. Grundy, "Improving automated documentation to code traceability by combining retrieval techniques," in ASE, 2011, pp. 223-232.
[6] W. Cohen, "Fast effective rule induction," in ICML, 1995.

[7] D. Cubranic and G. C. Murphy, "Automatic Bug Triage Using Text Categorization," in SEKE, 2004, pp. 92-97.

[8] P. Francis, D. Leon, and M. Minch, "Tree-based methods for classifying software failures," in ISSRE, 2004.

[9] M. Gegick, P. Rotella, and T. Xie, "Identifying security bug reports via text mining: An industrial case study," in MSR, 2010, pp. 11-20.

[10] S. Haiduc, J. Aponte, and A. Marcus, "Supporting program comprehension with source code summarization," in ICSE (2), 2010, pp. 223-226.

[11] P. Hooimeijer and W. Weimer, "Modeling bug report quality," in ASE, 2007, pp. 34-43.

[12] L. Huang, V. Ng, I. Persing, R. Geng, X. Bai, and J. Tian, "AutoODC: Automated generation of orthogonal defect classifications," in ASE, 2011.

[13] N. Jalbert and W. Weimer, "Automated duplicate detection for bug tracking systems," in DSN, 2008.

[14] A. Lamkanfi, S. Demeyer, E. Giger, and B. Goethals, "Predicting the severity of a reported bug," in MSR, 2010

[15] A. Lamkanfi, S. Demeyer, Q. Soetens, and T. Verdonck, "Comparing mining algorithms for predicting the severity of a reported bug," in CSMR, 2011

[16] C. D. Manning, P. Raghavan, and H. Schtze, Introduction to Information Retrieval. New York, NY, USA: Cambridge University Press, 2008, pp. 232-233.

[17] A. Marcus and J. I. Maletic, "Recovering documentation-to-sourcecode traceability links using latent semantic indexing," in ICSE, 2003, pp. 125-137.

[18] T. Menzies and A. Marcus, "Automated severity assessment of software defect reports," in ICSM, 2008.

[19] Www.openoffice.org/qa/ooQAReloaded/Docs/QA-ReloadedITguide.html\#priorities.

[20] A. Podgurski, D. Leon, P. Francis, W. Masri, M. Minch, J. Sun, and B. Wang, "Automated support for classifying software failure reports," in Proceedings of the 25th International Conference on Software Engineering, 2003, pp. 465-475.

[21] S. Robertson, H. Zaragoza, and M. Taylor, "Simple BM25 Extension to Multiple Weighted Fields," in Proceedings of the thirteenth ACM international conference on Information and knowledge management, 2004, pp. 42-49.

[22] P. Runeson, M. Alexandersson, and O. Nyholm, "Detection of duplicate defect reports using natural language processing," in ICSE, 2007, pp. 499-510.

[23] R. J. Sandusky, L. Gasser, and G. Ripoche, "Bug report networks Varieties, strategies, and impacts in a f/oss development community," in International Workshop on Mining Software Repositories, 2004, pp. $80-84$.

[24] G. Sridhara, L. L. Pollock, and K. Vijay-Shanker, "Automatically detecting and describing high level actions within methods," in ICSE, 2011, pp. 101-110.

[25] Www.ils.unc.edu/ keyeg/java/porter/PorterStemmer.java.

[26] C. Sun, D. Lo, S.-C. Khoo, and J. Jiang, "Towards more accurate retrieval of duplicate bug reports," in ASE, 2011.

[27] C. Sun, D. Lo, X. Wang, J. Jiang, and S.-C. Khoo, "A discriminative model approach for accurate duplicate bug report retrieval," in ICSE, 2010.

[28] A. Tamrawi, T. T. Nguyen, J. Al-Kofahi, and T. N. Nguyen, "Fuzzy set-based automatic bug triaging," in ICSE, 2011, pp. 884-887.

[29] A. Tamrawi, T. T. Nguyen, J. M. Al-Kofahi, and T. N. Nguyen, "Fuzzy set-based automatic bug triaging," in ICSE, 2011.

[30] M. Taylor, H. Zaragoza, N. Craswell, S. Robertson, and C. Burges, "Optimisation methods for ranking functions with multiple parameters," in Int. Conf. on Information and Knowledge Mgmt (CIKM), 2006.

[31] X. Wang, L. Zhang, T. Xie, J. Anvik, and J. Sun, "An approach to detecting duplicate bug reports using natural language and execution information," in ICSE, 2008, pp. 461-470.

[32] H. Zaragoza, N. Craswell, M. J. Taylor, S. Saria, and S. E. Robertson, "Microsoft cambridge at trec 13: Web and hard tracks," in TREC, 2004. 